\title{
On Mod 2 and Higher Elliptic Genera
}

\author{
Kefeng Liu \\ Department of Mathematics, Harvard University, Cambridge, MA 02138, USA
}

Received June 25, 1991; in revised form March 16, 1992

1. Abstract. In the first part of this paper, we construct mod 2 elliptic genera on manifolds of dimensions $8 k+1,8 k+2$ by $\bmod 2$ index formulas of Dirac operators. They are given by mod 2 modular forms or mod 2 automorphic functions. We also obtain an integral formula for the mod 2 index of the Dirac operator. As a by-product we find topological obstructions to group actions. In the second part, we construct higher elliptic genera and prove some of their rigidity properties under group actions. In the third part we write down characteristic series for all Witten genera by Jacobi theta-functions. The modular property and transformation formulas of elliptic genera then follow easily. We shall also prove that Krichever's genera, which come from integrable systems, can be written as indices of twisted Dirac operators for $S U$-manifolds. Some general discussions about elliptic genera are given.

\section{Introduction}

Elliptic genera were first constructed by Ochanine [28], Landweber-Stong in a topological way. Witten gave a geometric interpretation of them. More precisely, he showed that the Lefschetz fixed point formula of twisted Dirac operator on loop space gave the universal elliptic genera. Recently Krichever [20] derived certain elliptic genera from the theory of KP equations. Their constructions work for manifolds of dimension $8 k$ and $8 k+4$ or almost complex manifolds. Later Ochanine constructed mod 2 elliptic genera for manifolds of dimension $8 k+1$ and $8 k+2$ by cobordism theory in [29]. This construction was conjectured by Landweber [21].

In this paper, we construct mod 2 elliptic genera by the Atiyah-Singer mod 2 index formula. We take Witten's point of view to give a geometric construction of mod 2 elliptic genera. The method is quite easy. We lift the classical index formula to a $K O$-invariant version, and check that it is equal to the $K O$-invariant in cobordism theory. Hence we can borrow some beautiful ideas from cobordism theory. Theorem 1 and Corollary 1 answer a question of Witten in [36], viz. the 
mod 2 index invariants for each eigenvalue of $P$ (see [36]) sum up to a mod 2 modular form or to a $\bmod 2$ automorphic function. Theorem 2 is an integral formula for the mod 2 index, which answers a question of Atiyah (see [2]). Hopefully, a geometric understanding of our construction can eventually give a complete answer to Atiyah's question, i.e. a "good" integral formula for the mod 2 index. We also hope that this construction may indicate a way to prove the mod 2 index formula by an analytical method (see the discussion in the preface of [1]). As a simple corollary, we prove all invariants derived in [29] are spectral invariants. This includes the result in [30] as a special case. Also we will prove some vanishing theorems. These theorems show that our construction may give some new topological obstructions to group actions on compact smooth spin manifolds. We also put forward some questions about the geometric structure of the construction used in the proof of Theorem 1.

In the second part of this paper, we construct some twisted elliptic genera, and prove some of their rigidity properties. Then we consider higher elliptic genera, i.e. the elliptic genera twisted by representations of fundamental groups of manifolds. We also relate them to the higher Novikov conjecture. These results are closely related to the results in Browder-Hsiang [12], Berstein [10]. We follow the ideas of Atiyah-Hirzebruch [5] and Bott-Taubes [11]. It is interesting to notice that we prove some vanishing theorems of higher indices by the Lefschetz fixed point formula, and the topological condition naturally comes in. In a forthcoming paper, we will prove some vanishing theorems of secondary characteristic classes by combining our method with Conne's technique.

Then, by directly checking the geometric expressions of several elliptic genera of Witten, we write down the characteristic series for these genera. They are exactly the corresponding ratios or normalized ratios of Jacobi theta-functions. These expressions should be compared with the general Baker-Akhiezer functions in conformal field theory. From these simple expressions and transformation laws of theta-functions, several properties of elliptic genera follow immediately. For example, the modular property and the transformation law of different elliptic genera are natural consequences. Professor Landweber informed the author that $\mathrm{H}$. Tamanoi has also done this in his thesis. These expressions also indicate a way to construct other elliptic genera and a way to explain the Witten rigidity theorems of elliptic genera which we will discuss in a separate paper. What is also interesting here is that even the anomaly factor in Witten's formulas which came from renormalizations of Feymann path integrals perfectly fits our theta-function formulas. Another simple corollary is that we can interpret elliptic cohomology as a natural deformation of classical $\mathrm{KO}$-theory, or quantization of $\mathrm{KO}$-theory. In this part we shall also answer Krichever's question in [20], i.e. give index formula expressions of his genera which come from integrable systems. This is another motivation for us to study elliptic genera with theta-functions. By a simple manipulation in elliptic functions, we show that his genera give a continuous family of elliptic genera in which Hirzebruch's genera are included as a discrete family. But in many interesting cases these genera still can be expressed as in the indices of twisted Dirac operators and are rigid. So far all of the elliptic genera which are known to be rigid can be written as the indices of elliptic operators. It is interesting to speculate that all of the rigid genera should be the indices of some elliptic operators. We also note that the characteristic series of Krichever in Sect. 5 is basically the same as the function used in [38] by Zagier to get the generating function for Fourier coefficients of modular forms. 
Finally in the appendix we collect some known theorems in the theory of elliptic genera and give some general discussions. We give the formulas of generalized Chern character from elliptic cohomology to de Rham cohomology or $K$-theory. These formulas are quite similar to some partition functions in conformal field theory. Then as a simple application of the transformation formulas in Sect. 5 we prove a duality property between different elliptic genera. This was partially proved in [11] by a different argument. In a future paper we will study relations between various properties of the elliptic genera and the geometry of elliptic modular surfaces by using the results in Sect. 5.

Almost all of the results in this paper were obtained when the author was asked to report on Ochanine's paper [29] in Professor S.-T. Yau's seminar during the summer of 1990. The author would like to thank Professors Bott, Landweber, Witten and Yau for their kind help and encouragement. Both Professor Landweber and Professor Yau have carefully read several early versions of this paper and made many useful comments. Without them this paper would never have appeared in this form. The author is also very grateful to Professor Ochanine. His paper [29] motivated one crucial idea in this paper. In some sense this paper can be viewed as a simple step in trying to apply the beautiful elliptic genera theory which was developed by Landweber, Stong, Ochanine and Witten to quantum field theory and topology. Thanks are also due to Professor S. Stolz, a referee and the participants in Yau's seminar for their help in improving this paper. This research was partially supported by DOE grant DE-FG02-88ER-25065.

\section{Mod 2 Elliptic Genera}

Let $X$ be an $8 k+1$ or $8 k+2$ dimensional compact smooth spin manifold. Let $\Delta(X)=S^{0}(X) \oplus S^{1}(X)$ be the $\mathbf{Z}_{2}$-graded spinor bundle on $X$, and let

$$
D: S^{0}(X) \rightarrow S^{1}(X)
$$

be the Dirac operator on $X$. Let $E$ be any real vector bundle on $X$. Then we can form the twisted Dirac operator $D \otimes E$ and obtain skew adjoint or skew Hermitian elliptic operator, which gives
a) $\operatorname{dim}_{\mathrm{R}} \operatorname{Ker} D \otimes E \bmod 2$, if $\operatorname{dim} X=8 k+1$,
b) $\operatorname{dim}_{\mathbf{C}} \operatorname{Ker} D \otimes E \bmod 2$, if $\operatorname{dim} X=8 k+2$

as well defined indices which are topological invariants. We write them as Ind $_{2}(D \otimes E)$. These indices can be naturally extended to a homomorphism from $K O(X)$ to $\mathbf{Z}_{2}$ (see [7]). Now let

$$
\begin{aligned}
\Theta_{q}(E) & =\bigotimes_{n \geqq 1}\left(\Lambda_{-q^{\left(n-\frac{1}{2}\right)}} E \otimes S_{q^{n}} E\right) \\
\Theta_{q}^{*}(E) & =\bigotimes_{n \geqq 1}\left(\Lambda_{-q^{(2 n-1)}} E \otimes S_{q^{2 n}} E\right), \\
\Theta_{-q}(E) & =\bigotimes_{n \geqq 1}\left(\Lambda_{q^{\left(n-\frac{1}{2}\right)}} E \otimes S_{q^{n}} E\right) \\
\Theta_{q}^{\prime}(E) & =\bigotimes_{n \geqq 1}\left(\Lambda_{q^{n}} E \otimes S_{q^{n}} E\right)
\end{aligned}
$$


be elements in $K O(X) \llbracket q \rrbracket$, where

$$
\begin{aligned}
& \Lambda_{t} E=1+t E+t^{2} \Lambda^{2} E+\ldots, \\
& S_{t} E=\frac{1}{\Lambda_{-t} E}
\end{aligned}
$$

are two operations in $K O(X)$. Here we take $q=e^{2 \pi i \tau}$ with $\tau \in \mathbf{C}$ and $\operatorname{Im} \tau>0$.

These elements arose from quantum field theory (see [35]). We can expand them as formal power series of $q$. For example $\Theta_{q}(E)=\sum_{k \geqq 0}^{\infty} q^{k / 2} R_{k}$, then

$$
R_{0}=1, \quad R_{1}=E, \quad R_{2}=\Lambda^{2} E \oplus E, \quad R_{3}=\Lambda^{3} E \oplus(E \otimes E) \oplus E,
$$

which are the eigenbundles of the operator $P$ in quantum field theory for $E=T X$ (see Witten [35]). Here $T X$ is the tangent bundle of $X$. First we like to consider the virtual versions of the above elements. We take $E=T X-\operatorname{dim} X$ and expand them into the power series of $q$. For example

$$
\Theta_{q}(T X-\operatorname{dim} X)=\sum_{i \geqq 0} b_{i}(T X) q^{i / 2},
$$

where all $b_{i}$ 's are elements of $K O(X)$. In fact they are linear combinations of $R_{i}$ 's above (see [29]). Let us consider the virtual indices

$$
\operatorname{Ind}_{2}\left(D \otimes \Theta_{q}(T X-\operatorname{dim} X)\right)=\sum_{i \geqq 0} \operatorname{Ind}_{2}\left(D \otimes b_{i}(T X)\right) q^{i / 2} .
$$

Similarly for $\operatorname{Ind}_{2}\left(D \otimes \Theta_{q}^{*}(T X-\operatorname{dim} X)\right)$ and $\operatorname{Ind}_{2}\left(D \otimes \Theta_{-q}(T X-\operatorname{dim} X)\right)$. We will discuss the elliptic genera associated to $\Theta_{q}^{\prime}(T X-\operatorname{dim} X)$ later.

Let

$$
\begin{aligned}
\Gamma^{0}(2) & =\left\{\left(\begin{array}{ll}
a & b \\
c & d
\end{array}\right) \mid b \equiv 0(\bmod 2)\right\} \\
\Gamma_{0}(2) & =\left\{\left(\begin{array}{ll}
a & b \\
c & d
\end{array}\right) \mid c \equiv 0(\bmod 2)\right\} \\
\Gamma_{\theta} & =\left\{\left(\begin{array}{ll}
a & b \\
c & d
\end{array}\right) \mid\left(\begin{array}{ll}
a & b \\
c & d
\end{array}\right) \equiv\left(\begin{array}{ll}
1 & 0 \\
0 & 1
\end{array}\right) \text { or }\left(\begin{array}{ll}
0 & 1 \\
1 & 0
\end{array}\right)(\bmod 2)\right\}
\end{aligned}
$$

be three modular subgroups of $S L_{2}(\mathbf{Z})$.

Remember we call a holomorphic function $f(\tau)$ on the Poincaré upper half plane $\mathbf{H}$ a modular form of weight $k$ over a modular subgroup $\Gamma$ if for

$$
\begin{gathered}
\left(\begin{array}{ll}
a & b \\
c & d
\end{array}\right) \in \Gamma, \\
f\left(\frac{a \tau+b}{c \tau+d}\right)=(c \tau+d)^{k} f(\tau),
\end{gathered}
$$

and $f$ is holomorphic at the cusps of $\mathbf{H} / \Gamma$.

We denote the ring of modular forms over $\Gamma$ with integral Fourier coefficients by $M^{\mathrm{Z}}(\Gamma)$. From number theory (see [32]), we know that $M^{\mathrm{Z}}\left(\Gamma_{0}(2)\right.$ ) has an 
integral básis consisting of two elements (see below). As discussed in Sect. $5 \Gamma^{0}(2)$ and $\Gamma_{\theta}$ are conjugate to $\Gamma_{0}(2)$. So we can consider the modular forms with integral Fourier coefficients over these three modular groups modulo some prime number $p$ which are called $\bmod p$ modular forms (see [32]). Concerning the question of Witten in [35] we have:

Theorem 1. Let $X$ be a dimension $8 k+1$ or $8 k+2$ compact smooth spin manifold. Then the following mod 2 indices are mod 2 modular forms of weights $4 k$ over the corresponding modular groups

1) $\operatorname{Ind}_{2}\left(D \otimes \Theta_{q}(T X-\operatorname{dim} X)\right)$ over $\Gamma^{0}(2)$;

2) $\operatorname{Ind}_{2}\left(D \otimes \Theta_{q}^{*}(T X-\operatorname{dim} X)\right)$ over $\Gamma_{0}(2)$;

3) $\operatorname{Ind}_{2}\left(D \otimes \Theta_{-q}(T X-\operatorname{dim} X)\right)$ over $\Gamma_{\theta}$.

Proof. In the following we will identify our $\bmod 2$ indices as ring homomorphisms from $\Omega_{*}^{\text {spin }}$, the spin cobordism ring, to $M^{\mathrm{Z}}(\Gamma) \otimes K O^{*}(p t)$, the ring of modular forms over the modular group $\Gamma$ with coefficients in $K O^{*}(p t)$. Here $\Gamma$ denotes $\Gamma_{0}(2), \Gamma^{0}(2)$ or $\Gamma_{\theta}$. The proof is motivated by Ochanine's proof in [29]. We give the details and emphasize the analytical and the modular properties of these mod 2 indices.

We divide the proof into several steps.

Step 1. Consider the graded ring structure of $K O^{*}(p t)$; we know that it is generated by $\eta$, a degree 1 element, $\omega$, a degree 4 element, and $\mu$, a degree 8 element, over $\mathbf{Z}$ with relations

$$
2 \eta=\eta \omega=\eta^{3}=0, \quad \omega^{2}=4 \mu .
$$

Note that $K O^{*}(p t)$ has period 8, i.e. $K O^{* \pm 8}(p t) \simeq K O^{*}(p t)$ (see [4]). One has

$$
\begin{array}{ll}
K O^{-1}(p t)=\mathbf{Z}_{2} \eta, & K O^{-4}(p t)=\mathbf{Z} \omega, \\
K O^{-2}(p t)=\mathbf{Z}_{2} \eta^{2}, & K O^{-8}(p t)=\mathbf{Z} \mu .
\end{array}
$$

Consider the following Gysin homomorphism

$$
f_{1}: K O(X) \rightarrow K O^{-n}(p t),
$$

where $n=\operatorname{dim} X$, which is defined as follows: Consider an embedding.

$$
i: X \rightarrow S^{n+8 k}, \quad k \gg 0 \text {. }
$$

Let $N X$ be the normal bundle of $X$ inside $S^{n+8 k}, U(N X)$ be the $K O$-Thom class of $N X$. Then for any $E \in K O(X)$, we define:

$$
f_{!}(E)=j^{*}(E \cup U(N X)) \in \widetilde{K O}\left(S^{n+8 k}\right) \simeq K O^{-n}(p t),
$$

where $j$ is the natural isomorphism $D N / S N \simeq S^{8 k+n}$, and $D N, S N$ are respectively the disc bundle and sphere bundle of $N=N X$. We know $f_{!}$is independent of $i$.

Lemma 1. (KO-version of Atiyah-Singer mod 2 index formula [7]). Let $X$ be as above. Then for any $E \in K O(X)$,

$$
\operatorname{Ind}_{2}(D \otimes E) \equiv \pi\left(f_{!} E\right)(\bmod 2),
$$

where $\pi: K O^{-1}(p t) \simeq \mathbf{Z}_{2}, K O^{-2}\left(p_{2}\right) \simeq \mathbf{Z}_{2}$ are the isomorphisms sending $\eta$ and $\eta^{2}$ to $1 \in \mathbf{Z}_{2}$. 
Proof. This is implicit in the proof of Atiyah-Singer $\bmod 2$ index formula ([7]).

We define the mod $2 \mathrm{KO}$-index of an elliptic operator, written as $\mathrm{ind}_{2}$, as an element in $K O^{*}(p t)$. Then the above lemma tells us:

$$
\operatorname{ind}_{2}(D \otimes E)=f_{!}(E) \text {. }
$$

The following discussion will show that we have the similar $K O$-index formula for $8 k, 8 k+4$-dimensional manifolds as a lift of the classical index to $K O^{*}(p t)$. We will write them as ind.

Step 2. As a simple step, we will check the Gysin homomorphism defined above to be equal to the homomorphism defined in Stong [33] for $\mathrm{KO}$-characteristic classes of $X$. This seems to be well-known, but we cannot find a good reference. We prefer to give the details. Then we can borrow techniques in cobordism theory to prove Theorem 1. Recall the definitions in [33].

Define $K O$-characteristic classes $\pi_{i}(E)$, for a real vector bundle $E$ on $X$, as:

$$
\sum_{i \geqq 0} \pi_{i} \cdot u^{i}=\sum_{i \geqq 0} t^{i} \cdot \Lambda^{i}(E-\operatorname{dim} E),
$$

where $u=t /(1+t)^{2}$. Consider $X \in \Omega_{n}^{\text {spin }}$ as a homotopy class in $\pi_{n+8 k}\left(T B \operatorname{spin}_{8 k}\right)$, for $k$ big enough. Here $T B \operatorname{spin}_{8 k}$ is the Thom space of the universal bundle $\gamma_{8 k}$ over $\operatorname{Bspin}_{8 k}$. And let

$$
f_{X}: S^{n+8 k} \rightarrow T B \operatorname{spin}_{8 k}
$$

be the representative of $X$. We define the $K O$-characteristic numbers $\pi_{i}(X)$ corresponding to $\pi_{i}(T X)$ as

$$
\pi_{i}(X)=f_{X !}\left(\pi_{i}(T X)\right)=f_{X}^{*}\left(\pi_{i} \cup U \gamma_{8 k}\right) \in \widetilde{K O}\left(S^{n+8 k}\right) \simeq K O^{-n}(p t),
$$

where $U \gamma_{8 k}$ is the Thom class of $\gamma_{8 k}$ and $\pi_{i}=\pi_{i}\left(\gamma_{8 k}\right)$.

By Thom construction, we know that the pull-back of the zero section of $T B \operatorname{spin}_{8 k}$ by $f_{X}$ is the manifold $X$ and the pull-back of $\gamma_{8 k}$ is $N X$. From this description and the naturality of Gysin homomorphism, we know that if we take the pull-back of the zero section of $T B \operatorname{spin}_{8 k}$ as our embedding, then the above two Gysin homomorphisms coincide for $\pi_{i}(T X) \in K O(X)$. So we have:

Lemma 2. $\operatorname{ind}_{2}\left(D \otimes \pi_{i}(T X)\right)=\pi_{i}(X)$.

So we have proved that the indices of the Dirac operator twisted by $\mathrm{KO}$ characteristic classes are cobordism invariants.

If we write $\Theta_{q}(E-\operatorname{dim} E)=\sum_{i \geqq 0} b_{i} q^{i / 2}$ by expressing:

$$
\Theta_{q}(E-\operatorname{dim} E)=\bigotimes_{n \geqq 1}\left(\Lambda_{-q^{n-\frac{1}{2}}}(E-\operatorname{dim} E) / \Lambda_{-q^{n}}(E-\operatorname{dim} E)\right)
$$

and expand it as the formal power series in $q$, we can easily see that every $b_{i}$ is a polynomial in $\pi_{i}(E)$. For example $b_{0}=1, b_{1}=-\pi_{1}$, etc. So we have

$$
\operatorname{ind}_{2}\left(D \otimes \Theta_{q}(T X-\operatorname{dim} X)\right)=f_{X !}\left(\Theta_{q}(T X-\operatorname{dim} X)\right),
$$

where $f_{X !}$ is the Gysin homomorphism in Step 2, which is the same as $f_{!}$in Step 1 by the above discussion. 
Step 3. Now we lift the Landweber-Stong-Witten construction of elliptic genera $8 k$, for $8 k+4$ manifold to $K O$-invariants. We first consider 1 ).

For an $8 k$, or $8 k+4$ manifold $X$, we have the elliptic genera:

$$
\begin{aligned}
\operatorname{Ind}\left(D \otimes \Theta_{q}(T X-\operatorname{dim} X)\right) & =\int_{X} \hat{A}(X) \operatorname{ch}\left(\Theta_{q}(T X-\operatorname{dim} X) \otimes \mathbf{C}\right) \\
& \in M^{\mathbf{Z}}\left(\Gamma^{0}(2)\right) \subset \mathbf{Z}\left[\delta^{0}, \varepsilon^{0}\right],
\end{aligned}
$$

where $D$ is the pure Dirac operator on $X$, and $\delta^{0}$ and $\varepsilon^{0}$ are two integral generators of the ring $M^{\mathbf{Z}}\left(\Gamma^{0}(2)\right)$. Note that the elliptic genera we constructed here are slightly different from those in [21]. So we get modular forms over $\Gamma^{0}(2)$ instead of $\Gamma_{0}(2)$. The integral property of these genera come from their expressions as the indices of an elliptic operator. The modular property will be discussed in Sect. 5 of this paper.

We then use the Atiyah-Hirzebruch-Riemann-Roch theorem for differentiable manifolds to get the following commutative diagram:

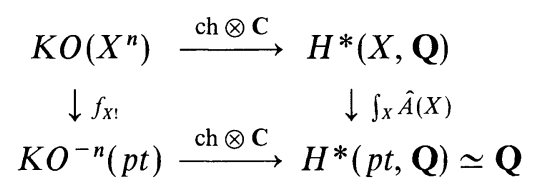

and on $K O^{*}(p t)$, we have

$$
\operatorname{ch}(\eta \otimes \mathbf{C})=0, \quad \operatorname{ch}(\omega \otimes \mathbf{C})=2, \quad \operatorname{ch}(\mu \otimes \mathbf{C})=1
$$

(see [4]).

By a similar discussion as in Landweber [21], we know that $\left.\int_{X} \hat{A}(X) \operatorname{ch}\left(\Theta_{q}(T X-\operatorname{dim} X)\right) \otimes \mathbf{C}\right)=P\left(\delta^{0}, \varepsilon^{0}\right) \in \mathbf{Z}\left[\delta^{0}, \varepsilon^{0}\right]$, where $P\left(\delta^{0}, \varepsilon^{0}\right)$ is a modular form of weight $4 k$ or $4 k+2$ depending on whether the dimension of $X$ is $8 k$ or $8 k+4$ respectively. (From now on we will first restrict ourself to $8 k+2$ manifolds, $8 k+1$ case will be discussed later.) In fact, $P\left(\delta^{0}, \varepsilon^{0}\right)$ is a weighted homogeneous integral polynomial in $\delta^{0}$ and $\varepsilon^{0}$. The weight of $\delta^{0}$ is 2 , the weight of $\varepsilon^{0}$ is 4 . So we must have

$$
f_{X !}\left(\Theta_{q}(T X-\operatorname{dim} X)\right)=\frac{1}{2} P\left(\delta^{0}, \varepsilon^{0}\right) \omega
$$

because $K O^{-4}(p t)=\mathbf{Z} \omega$ and $\operatorname{ch}(\omega \otimes \mathbf{C})=2$. Since $P\left(\delta^{0}, \varepsilon^{0}\right)$ always has even coefficients for $8 k+4$-manifolds, we get our $K O$-index elliptic genera for dimension $8 k+4$ manifolds,

$$
\operatorname{ind}\left(D \otimes \Theta_{q}(T X-\operatorname{dim} X)\right) \in \mathbf{Z}\left[\delta^{0}, \varepsilon^{0}\right] \omega .
$$

Step 4. Now we use a construction from cobordism theory to prove our theorem, i.e. for $8 k+2$-dimensional manifold, $\operatorname{ind}_{2}\left(D \otimes \Theta_{q}(T X-\operatorname{dim} X)\right)$ is a $\bmod 2$ modular form. This contruction is due to Stong [33] and used by Ochanine in [29].

Following Ochanine and Stong, for any $X \in \Omega_{8 k+2}^{\text {spin }}$, we construct a manifold $T(X) \in \Omega_{8 k+4}^{\text {spin }}$. Recall that $K O$-invariants are spin cobordism invariants, so our indices are also spin cobordism invariants.

Since $X \times \bar{S}^{1} \in \Omega_{8 k+3}^{\text {spin }}=0$, we have $X \times \bar{S}^{1}=\partial W$ for a spin manifold $W$. Here $\bar{S}^{1}$ is $S^{1}$ with the nontrivial spin structure. As $2 \bar{S}^{1}=0$ in $\Omega_{1}^{\text {spin }}, 2 \bar{S}^{1}=\partial D$. We construct $T(X)=(-2 W) \coprod(X \times D)$, by gluing along the common boundary $X \times 2 \bar{S}^{1}$. This is a well defined homomorphism from $\Omega_{8 k+2}^{\text {spin }}$ to $\Omega_{8 k+4}^{\text {spin }} \otimes \mathbf{Z}_{2}$ (see 
Stong [33]). In fact, let $I_{*}$ be the ideal in $\Omega_{*}^{\text {spin }}$ which consists of elements with vanishing $K O$-characteristic numbers, i.e. for $X \in I_{*}$, all $\pi_{I}(X)=f_{X !}\left(\pi_{I}(T X)\right)=0$ for any $\pi_{I}=\pi_{i_{1}} \pi_{i_{2}} \ldots \pi_{i_{\alpha}}$. Then we have

Lemma 3 (Stong [33], Ochanine [29]): $T: \Omega_{8 k+2}^{\mathrm{spin}} / I_{8 k+2} \rightarrow \Omega_{8 k+4}^{\mathrm{spin}} / I_{8 k+4} \otimes \mathbf{Z}_{2}$ is an isomorphism, and the following diagram is commutative,

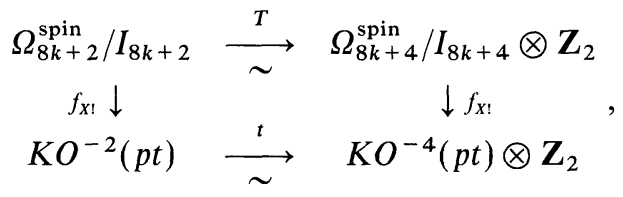

where $t$ is the isomorphism mapping $\eta^{2}$ to $\omega \otimes 1 \in K O^{-4}(p t) \otimes \mathbf{Z}_{2}$, i.e. we have

$$
\pi_{i}(T(V)) \otimes 1=t\left(\pi_{i}(V)\right) \text { for } V \in \Omega_{8 k+2}^{\mathrm{spin}} / I_{8 k+2} \text {. }
$$

Let

and

$$
\varphi_{q}(X)=\operatorname{ind}\left(D \otimes \Theta_{q}(T X-\operatorname{dim} X)\right) \quad \text { for } X \in \Omega_{8 k+4}^{\mathrm{spin}}
$$

Then

$$
\varphi_{q}(X)=\operatorname{ind}_{2}\left(D \otimes \Theta_{q}(T X-\operatorname{dim} X)\right) \text { for } X \in \Omega_{8 k+2}^{\text {spin }} .
$$

$$
\varphi_{q}(T(X)) \otimes 1=t\left(\varphi_{q}(X)\right) \quad \text { in } K O^{-4}(p t) \otimes \mathbf{Z}_{2} .
$$

It is easy to see that

$$
\varphi_{q}(T(X))=P\left(\delta^{0}, \varepsilon^{0}\right) \omega .
$$

Therefore we have that

$$
\varphi_{q}(X)=\eta^{2} P\left(\delta^{0}, \varepsilon^{0}\right)=\eta^{2} P\left(\bar{\delta}^{0}, \bar{\varepsilon}^{0}\right)
$$

is a mod 2 modular form (or modular form modulo 2 by the definition) where $\bar{\delta}^{0}, \bar{\varepsilon}^{0}$ are the modulo 2 reduction of $\delta^{0}$ and $\varepsilon^{0}$. This finishes the proof of Theorem 1 for $8 k+2$ dimensional case.

Now let us prove Theorem 1 for $8 k+1$ dimensional manifolds. We can consider $X \times \bar{S}^{1}$ for $X \in \Omega_{8 k+1}^{\text {spin }}$. We have

$$
\varphi_{q}\left(X \times \bar{S}^{1}\right)=\varphi_{q}(X) \cdot \eta .
$$

Since the multiplication by $\eta$ is an isomorphism from $\mathrm{KO}^{-1}(p t)$ to $\mathrm{KO}^{-2}(p t)$ and $\varphi_{q}\left(X \times \bar{S}^{1}\right)$ is a mod 2 modular form by the result for $8 k+2$ case, $\varphi_{q}(X)$ is also a mod 2 modular form.

For another proof we can consider the multiplication by $\bar{S}^{1}$ which gives a morphism,

$$
S: \Omega_{8 k}^{\text {spin }} \rightarrow \Omega_{8 k+1}^{\text {spin }} .
$$

Then it is easy to verify that $\pi_{i}\left(\bar{S}^{1} \times M\right)=\eta \cdot \pi_{i}(M)$ for any spin manifold $M$ of dimension $8 k$. By Stong [33] $S$ induces a surjective homomorphism

$$
\tilde{S:} \Omega_{8 k}^{\text {spin }} / I_{8 k} \rightarrow \Omega_{8 k+1}^{\text {spin }} / I_{8 k+1} .
$$

It follows that

$$
\varphi_{q}\left(\Omega_{8 k+1}^{\mathrm{spin}}\right)=\eta \cdot \varphi_{q}\left(\Omega_{8 k}^{\mathrm{spin}}\right)
$$


In other words, for an $8 k+1$ dimensional manifold $X, \varphi_{q}(X)$ is the modulo 2 reduction of the elliptic genus of some dimension $8 k$ manifold. So it is a mod 2 modular form. This finishes the proof of 1) of Theorem 1.2) and 3) can be proved in the same way. Note that the modular properties follow from the theta-function expressions of the elliptic genera in Sect. 5.

The construction in the second proof of Theorem 1 for $8 k+1$ dimensional case is also due to Stong and used by Ochanine in [29].

Recall that we call a holomorphic function $f(\tau)$ on the upper half plane a (twisted) automorphic function over a modular group $\Gamma$ if it satisfies the following transformation law,

$$
f\left(\frac{a \tau+b}{c \tau+d}\right)=\chi(\gamma) f(\tau)
$$

for

$$
\gamma=\left(\begin{array}{ll}
a & b \\
c & d
\end{array}\right) \in \Gamma,
$$

where $\chi$ is a character from $\Gamma$ to some finite group in $\mathbf{C}^{*}$. If $f$ has integral Fourier coefficients we can consider its modulo 2 reduction which we call the mod 2 automorphic function.

By combining the above proof and the discussion in Sect. 5 we can get the following corollary which is the non-virtual version of Theorem 1.

Corollary 1. The following mod 2 indices are (twisted) $\bmod 2$ automorphic functions over the corresponding modular groups

1) $q^{-k / 2} \operatorname{Ind}_{2} D \otimes \Theta_{q}(T X)$ over $\Gamma^{0}(2)$;

$2 q^{-k / 2} \operatorname{Ind}_{2} D \otimes \Theta_{-q}(T X)$ over $\Gamma_{\theta}$;

$3 q^{-k} \operatorname{Ind}_{2} D \otimes \Theta_{q}^{*}(T X)$ over $\Gamma_{0}(2)$.

In fact the automorphic functions in the above corollary are twisted by some characters from those modular groups to the group $\{ \pm i, \pm 1\}$.

For

$$
X, Y \in \Omega_{*}^{\text {spin }},
$$

we have

$$
\Theta_{q}(T(X \times Y)-\operatorname{dim}(X \times Y))=\Theta_{q}(T X-\operatorname{dim} X) \otimes \Theta_{q}(T Y-\operatorname{dim} Y) .
$$

Similar formulas are also true for $\Theta_{-q}(T X-\operatorname{dim} X)$ and $\Theta_{q}^{*}(T X-\operatorname{dim} X)$.

So if we let $\varphi_{q}(X)=\operatorname{ind}\left(D \otimes \Theta_{q}(T X-\operatorname{dim} X)\right)$ for $\operatorname{dim} X=8 k$ or $8 k+4$, and $\varphi_{q}(X)=\operatorname{ind}_{2}\left(D \otimes \Theta_{q}(T X-\operatorname{dim} X)\right)$ for $\operatorname{dim} X=8 k+1$ or $8 k+2$. We define $\psi_{q}(X)$ and $\phi_{q}(X)$ for the elliptic genera associated to $\Theta_{-q}(T X-\operatorname{dim} X)$ and $\Theta_{q}^{*}(T X-\operatorname{dim} X)$ respectively in the same way. Then

$$
\begin{aligned}
& \varphi_{q}(X \times Y)=\varphi_{q}(X) \cdot \varphi_{q}(Y) ; \\
& \phi_{q}(X \times Y)=\phi_{q}(X) \cdot \phi_{q}(Y) ; \\
& \psi_{q}(X \times Y)=\psi_{q}(X) \cdot \psi_{q}(Y) .
\end{aligned}
$$

By combining the above discussion and the results in Sect. 5 we have 
Corollary 2. We have the following graded ring homomorphisms

1) $\varphi_{q}: \Omega_{*}^{\text {spin }} \rightarrow K O^{*}(p t) \otimes M^{\mathbf{Z}}\left(\Gamma^{0}(2)\right)$;

2) $\psi_{q}: \Omega_{*}^{\text {spin }} \rightarrow K O^{*}(p t) \otimes M^{\mathbf{Z}}\left(\Gamma_{\theta}\right)$;

3) $\phi_{q}: \Omega_{*}^{\text {spin }} \rightarrow K O^{*}(p t) \otimes M^{\mathbf{Z}}\left(\Gamma_{0}(2)\right)$.

Here the grading on $\Omega_{*}^{\text {spin }}$ is given by the dimensions of manifolds. The grading on $K O^{*}(p t)$ is the degrees of the elements $\{\eta, \omega, \mu\}$. The grading on $M^{\mathrm{Z}}(\Gamma)$ is given by the weights of its elements. The grading on $K O^{*}(p t) \otimes M^{\mathbf{Z}}(\Gamma)$ is the sum of the gradings on $K O^{*}(p t)$ and $M^{\mathrm{z}}(\Gamma)$. Note the periodicity of $K O^{*}(p t)$.

From number theory [32] we know that $M^{\mathrm{Z}}\left(\Gamma_{0}(2)\right)$ is generated by two elements which we denote by $\delta_{0}, \varepsilon_{0}$. Then

$$
\begin{aligned}
& \delta_{0}=-1-24 \sum_{n \geqq 1}\left(\sum_{\substack{d \mid n \\
d \text { odd }}} d\right) q^{n}, \\
& \varepsilon_{0}=\sum_{n \geqq 1}\left(\sum_{\substack{d \mid n \\
n / d \text { (odd) }}} d^{3}\right) q^{n} .
\end{aligned}
$$

Obviously

$$
\bar{\delta}_{0} \equiv 1(\bmod 2)
$$

Since $\Gamma_{\theta}$ and $\Gamma^{0}(2)$ are conjugate to $\Gamma_{0}(2)$, the rings of integral modular forms over them are also conjugate to $M^{\mathbf{Z}}\left(\Gamma_{0}(2)\right)$ by the transformations $S$ and $S T$ respectively (see Sect. 5). If we replace $\tau$ by $2 \tau$ then $\varphi_{q}$ is changed to $\phi_{q}$. The change of the modular properties can be easily seen by the formulas in Sect. 5. Similarly if we change $\tau$ to $\tau+1 \varphi_{q}$ is changed to $\psi_{q}$.

Presumably $\operatorname{Ind}_{2}\left(D \otimes \Delta(X) \otimes \Theta_{q}^{\prime}(T X-\operatorname{dim} X)\right)$ should come into play. But this genus is not a "good" one for spin cobordism. With slight modification this genus can be defined for non-spin manifolds. We conjecture that this genus is a mod 2 modular form for any (non-spin) manifold $X$ of dimension $4 k+1$.

Now we consider the cohomology theory induced by $\phi_{q}$. Similar results are also true for the other two elliptic genera.

When $q=0$, we have $\phi_{q}(X)=\hat{\mathfrak{U}}(X)$ where

$$
\hat{\mathfrak{U}}(X)= \begin{cases}\operatorname{ind}_{2} D & X \text { has dimension } 8 k+1 \text { or } 8 k+2 \\ \operatorname{ind} D & X \text { has dimension } 8 k+4 \text { or } 8 k\end{cases}
$$

is the classical $\hat{\mathfrak{U}}$-invariant and we know

$$
K O_{*}(X)=\Omega_{*}^{\text {spin }}(X) \otimes_{\Omega_{*}^{\text {spin }}} K O_{*}(p t),
$$

where we use $K O_{*}(p t)$ to replace $K O^{*}(p t)$ as suggested by Landweber. We know they are isomorphic (see [18]). We consider $K O_{*}(p t)$ as a $\Omega_{*}^{\text {spin }}$ modulue through $\hat{\mathfrak{U}}=$ ind $D$ or $\operatorname{ind}_{2} D$.

Hence it is natural to consider the refined elliptic homology (see Hovey [19])

$$
\mathrm{Ell}_{*}(X)=\Omega_{*}^{\mathrm{spin}}(X) \otimes_{\Omega_{*}^{\text {spin }}}\left(K O_{*}(p t) \otimes M^{\mathrm{Z}}\left(\Gamma_{0}(2)\right)\left[\left(64\left(\delta_{0}^{2}-\varepsilon_{0}\right)\right)^{-1}\right]\right.
$$

as a "quantization" of $K O_{*}(X)$ in some sense. Here we view $K O_{*}(p t) \otimes M^{\mathrm{z}}\left(\Gamma_{0}(2)\right)$ as a $\Omega_{*}^{\text {spin }}$ module through $\phi_{q}$ above. This point of view is more interesting if we 
look at the projective schemes of the above graded rings. For example for a compact Lie group $G$ it tells us that the elements in Ell* $(B G)$ should be sections of a sheaf on the modular curve of $\Gamma_{0}(2)$. We do not pursue this point here.

For a manifold $X$ we know $K^{*}(X)=K_{*}\left(C_{0}(X)\right)$, where $C_{0}(X)$ is the algebra of continuous functions on $X$ with compact support. Can we deform $C_{0}(X)$ to get a new algebra $C_{0}(X)[h]$ as people are doing in quantum groups, such that

$$
K_{*}\left(C_{0}(X)[h]\right)=\operatorname{Ell}^{*}(X)
$$

with $q=e^{i n}$ ? This is another reason that we consider elliptic cohomology as "quantization of $\mathrm{KO}$-theory."

By Serre [32], we know that $\bar{\varepsilon}_{0}$ is a generator of the ring of mod 2 modular forms for $\Gamma_{0}(2)$.

Consider $\phi_{q} \bmod 2$, and write

$$
\phi_{q}(X) \equiv a_{0}+a_{1} \bar{\varepsilon}_{0}+\cdots+a_{m} \bar{\varepsilon}_{0}^{m}(\bmod 2)
$$

in $K O^{*}(p t) \otimes \mathbf{Z}_{2}\left[\bar{\varepsilon}_{0}\right]$, where $m=2 k$ for $\operatorname{dim} X=8 k+s, 0 \leqq s \leqq 4$, and $a_{i} \in K O^{*}$ $(p t)$. We have

Corollary 3. All the $a_{i}^{\prime} s$ above are spectral invariants. This is, in particular, true for the KO-Brown-Kervaire invariant. If $X$ admits a metric with positive scalar curvature, $\phi_{q}(X) \equiv \bar{\varepsilon}_{0}\left(a_{1}+\cdots+a_{m} \bar{\varepsilon}_{0}^{m-1}\right)(\bmod 2)$.

Proof. By Ochanine [29], we know that the so-called KO-Brown-Kervaire invariant is defined by

$$
\mathfrak{f}\left(V^{n}\right)= \begin{cases}\sigma(V) \mu & n \equiv 0(\bmod 8) \\ k\left(\bar{S}^{1} \times V\right) \eta & n \equiv 1(\bmod 8) \\ k(V) \eta^{2} & n \equiv 2(\bmod 8) \\ (\sigma(V) / 16) \omega & n \equiv 4(\bmod 8)\end{cases}
$$

modulo 2. Here $\sigma(V)$ is the signature and $k(V)$ is defined as $k(V) \equiv \sigma(U) / 8(\bmod 2)$ where $\partial U=\bar{S}^{1} \times V$. It is equal to $a_{m}$ above. The second statement follows from $\phi_{0}(X)=a_{0}=\operatorname{ind}_{2} D$.

Here by spectral invariant we mean that this invariant can be derived from elliptic operators. For example the index of an elliptic operator, analytic torsion and $\eta$-invariant are spectral invariants (see [8]).

The proof of the following Theorem 2 is a direct corollary of the proof of Theorem 1. But considering the discussions in Atiyah [1,2 and 7], we would like to single it out as a theorem and will study it in another paper.

Theorem 2. For $X$ a compact $8 k+2$ dimensional smooth spin manifold, let $T(X)$ be constructed as above. Then

$$
\operatorname{Ind}_{2} D=\hat{\mathfrak{A}}(X) \equiv \frac{1}{2} \int_{T(X)} \hat{A}(T(X))(\bmod 2) .
$$

In fact, we can get a similar formula for a dimension $8 k+1$ manifold by crossing $X$ by $\bar{S}^{1}$, and also we can get a general formula by twisting $D$ by $\pi_{i}(X)$ 's 
above. It is also possible to get formulas for $D$ twisted by general real vector bundles.

Mod 2 index is a very subtle invariant (see $[2,7]$ ). This is because of the absence of a cohomological formula as in $8 k$ and $8 k+4$ dimensional cases. The above Theorem 2 gives us a cohomological formula. But because the relation between the geometric structure of $X$ and that of $T(X)$ is not very clear, it is still difficult to handle it. It may be interesting to study the relation between the mod 2 indices of $X$ and those of $T(X)$ by using $\eta$-invariants which appeared in the construction of [8].

Considering the discussions in Atiyah $[1,2]$, this integral formula deserves further study. Its topological meaning is very simple. In fact, it is nothing but the Toda bracket in homotopy theory. But a geometric understanding of this formula may be useful for the computation of mod 2 index and an analytical proof of mod 2 index formula.

Instead of proving this theorem we would like to explain the homotopic background of the above $T$-construction. Let $f: S^{n+8 k} \rightarrow T B \operatorname{spin}_{8 k}$ represent $M$, $h: S^{n+8 k+1} \rightarrow S^{n+8 k}$ the suspension of the Hopf map, and 2: $S^{n+8 k+1} \rightarrow S^{n+8 k+1}$ a degree 2 map. We deform 2 and $h$ to be transverse regular on every point other than the base point, the inverse image of any regular value of $h \circ 2$ in $S^{n+8 k}$ being $2 \bar{S}^{1}$. If $f$ is also transverse regular on $B \operatorname{spin}_{8 k}$ then $f \circ h \circ 2$ is transverse regular on $B \operatorname{spin}_{8 k}$ and defines the spin manifold $2 \bar{S}^{1} \times M$. Considering the cobordism $U, V$ as given by maps $u: D^{n+8 k+1} \rightarrow T B \operatorname{spin}_{8 k}$ and $v: D^{n+8 k+2} \rightarrow S^{n+8 k}(V$ being a framed cobordism of $2 \bar{S}^{1}$ to zero) extending $f \circ h$ and $h \circ 2$ respectively. $u \circ 2, f \circ v$ fit together along their boundary to define a map of $n+8 k+2$ sphere into $T B$ spin $_{8 k}$ which represents $T(M)$. For more detail see Stong [33].

As a simple application of Theorem 2, we have

Corollary 4. Suppose there exists an $S^{1}$ action on an $8 k+2$ dimensional compact spin manifold $X$, and this action can be extended to $T(X)$ above, then

$$
\operatorname{Ind}_{2} D=\hat{\mathfrak{A}}(X)=0 .
$$

In fact, we only need that this $S^{1}$ action can be extended to $W$, where $\partial W=X \times \bar{S}^{1}$ as above.

Lawson-Yau [22] proved that an $S U(2)$ or $S O(3)$ action on an compact spin manifold leads to the vanishing of $\hat{\mathfrak{U}}$. We also note that there exists a homology sphere of type $M \times S^{1}$ and dimension $8 k+2$ with $\hat{\mathfrak{U}} \neq 0$ (see Milnor [24]). Also, Ochanine proved that an odd type $S^{1}$-action on an $8 k+1$ or $8 k+2$ dimensional manifold leads to the vanishing of the elliptic genera. He also conjectured that elliptic genera are all of the obstructions to the existence of an odd type $S^{1}$-action on an $8 k+1$ or $8 k+2$ dimensional manifold. Stolz's result [34] may be useful to prove this conjecture.

In many cases, we can draw some information of elliptic genera by considering the corresponding elliptic operators. For example, Theorem 1 together with Corollary 1 (for $\varphi_{q}$ ) completely answers a question of Witten in [35], which was derived from quantum field theory and formulated in terms of analytic indices. We can also get some vanishing theorems of the above elliptic genera under certain group action. For $8 k+1$ manifolds we have proved that the existence of an odd type involution results in the vanishing of their mod 2 elliptic genera. We would like to give the details of this discussion as well as some other results in a separate paper. In that paper we will also study some vanishing properties of the above elliptic genera when there exist vector fields on the manifold. 
It is interesting to study the relations between the elliptic genera in Theorem 1 and Corollary 1 through elliptic modular surfaces over $\mathbf{Z}_{2}$. See the discussion in Sect. 5 for the classical cases.

\section{Higher Elliptic Genera}

We still let $D$ be the pure Dirac operator on a compact smooth spin manifold $X$. Let $G$ be a compact Lie group acting on $X$. For a complex vector bundle on $X$ we say that $G$ acts on $E$ trivially if the $G$ action can be lifted onto $E$ and $E$ is equivariantly trivial on each $G$ orbit. For simplicity we assume that $G$ is $S^{1}$ in this section. But the results in this section are still true for general connected compact Lie groups. When $G$ is $S^{1}$ and acts trivially on $E$, the induced action of $G$ on $E$ restricted to the fixed points of $G$ is trivial, i.e. the exponents of a generator of $G$ are zero. See below for the definition of exponent. We take Ind $\left(D \otimes \Theta_{q}(T X-\operatorname{dim} X)\right)$ as an example. The same conclusions hold for the other elliptic genera discussed in Sect. 5.

Theorem 3. Let $X$ be a connected compact smooth spin manifold with an $S^{1}$ action. Let $E$ be a complex vector boundle on $X$. Assume the $S^{1}$ acts on $E$ trivially, then

a) $\operatorname{Ind}(D \otimes E)=0$;

b) $\operatorname{Ind}\left(D \otimes \Theta_{q}(T X-\operatorname{dim} X) \otimes E\right)$ is rigid.

Remember we call an index rigid if its corresponding Lefschetz numbers are constant as a character on the action group.

Proof. Let $g$ be a generator of the $S^{1}$ actioon and $L\left(D_{E}, g\right)$ be the Lefschetz number of $g$. Then we have the Atiyah-Segel-Singer fixed point theorem (see [6])

$$
\left.L\left(D_{E}, g\right)=\sum_{\left\{F_{g}\right\}} \text { (local term on } F_{g}\right),
$$

where $\left\{F_{g}\right\}$ are the fixed point submanifolds of $g$. The normal bundle $N$ to $F_{g}$ has an $S^{1}$ invariant decomposition

$$
N=\bigoplus_{m \in \mathbf{Z}} N_{m},
$$

where each $N_{m}$ carries the structure of a complex bundle and $g$ acts on $N_{m}$ by scalar multiplication by $t^{m}=e^{i m \theta}$. The $m$ 's are called the exponents of $g$.

For any given $k$-dimensional complex vector bundle $N$, we define the function

$$
F(N, t)=\prod_{j=1}^{t} \frac{1}{t^{\frac{1}{2}} e^{\frac{1}{2} x_{j}}-t^{-\frac{1}{2}} e^{-\frac{1}{2} x_{j}}},
$$

where $x_{j}$ 's are the formal roots of the total Chern class of $N$. If the action is trivial on the given bundle $E$, up to sign the local term above can be reduced to

$$
\prod_{m} F\left(N_{m}, t^{m}\right) \hat{A}\left(F_{g}\right) \operatorname{ch}\left(i_{g}^{*} E\right)\left[F_{g}\right],
$$

where $i_{g}: F_{g} \hookrightarrow M$ is the inclusion map. On the other hand since the Lefschetz number is the character of $g$ acting on a finite dimensional vector space,

$$
L\left(D_{E}, g\right)=\sum_{k=-N}^{N} n_{k} t^{k}
$$


is a Laurent polynomial. It is evident that the local expression in the fixed point formula is zero for $t=0$ and $t=\infty$ and the Laurent polynomial expression can only have poles at $t=0$ and $t=\infty$. Hence $L\left(D_{E}, g\right)$ must vanish identically. Then note that when $t=1 L\left(D_{E}, g\right)$ is $\operatorname{Ind}(D \otimes E)$.

For elliptic genera, we only need to note that if the group action on $E$ is trivial, the crucial trick, "transfer formula," in Bott-Taubes [11] still works. We omit the details here.

Corollary 5. Let $X$ be as in Theorem 4. Assume the induced $S^{1}$-action on $\pi_{1}(X)$ is trivial. Then

a) $\operatorname{Ind}(D \otimes E)=\langle\hat{A}(X) \operatorname{ch} E, X\rangle=0$,

b) $\operatorname{Ind}\left(D \otimes \Theta_{q}(T X-\operatorname{dim} X) \otimes E\right)=\left\langle\hat{A}(X) \operatorname{ch} \Theta_{q}(T X-\operatorname{dim} X) \operatorname{ch} E, X\right\rangle$ is rigid, where $E$ is a flat (infinite dimensional) vector bundle associated to a unitary representation of $\pi_{1}(X)$, and our index and Chern character are in the sense of Mischenko-Fomenko [25].

Proof. What we need to do is to carefully define the Lefschetz number for the elliptic operator in Mischenko-Fomenko's category (see [25]) and show that the fixed point formula is true in this case.

Let $A$ be a $C^{*}$-algebra with unit and $G$ be a compact Lie group. Assume there is no action of $G$ on $A$. Let $D_{E}$ be an elliptic operator twisted by an $A$-module vector bundle $E$ (see [31]). Since $G$ is compact, we can assume $G$ commutes with $D_{E}$. Then for some $G$-invariant compact operator $K \operatorname{Ker}\left(D_{E}+K\right)$ and $\operatorname{Coker}\left(D_{E}+K\right)$ are $A$-modules of finite rank with $G$-actions (see [25 or 31$]$ ). We define

$$
\operatorname{Ind}_{G}\left(D_{E}\right)=\operatorname{Ker}\left(D_{E}+K\right)-\operatorname{Coker}\left(D_{E}+K\right) \in K_{G}(A) \simeq K_{0}(A) \otimes R(G)
$$

as our $G$-index with value in $K_{0}(A) \otimes R(G)$. Here $K_{G}(A)$ denotes the $K$-group of $G$-equivalent $A$-modules. Since there is no $G$ action on $A$,

$$
K_{G}(A) \simeq K_{0}(A) \otimes R(G) .
$$

For $g \in G$, let

$$
\operatorname{Ind}_{G}\left(D_{E}\right)(g) \in K_{0}(A)[\chi(g)]
$$

denote the Lefschetz number of $g$ which is obtained from $\operatorname{Ind}_{G}\left(D_{E}\right)$ by taking trace on the $R(G)$-part of $K_{G}(A)$. Here $\chi(g)$ denotes the character of $g$ and $K_{0}(A)[\chi(g)]$ is the polynomial ring in $\chi(g)$ with coefficients in $K_{0}(A)$. Then we have the following

Lemma 4. Let $X$ be a d-dimensional compact smooth spin manifold with an $S^{1}$ action. Let $D_{E}$ be an elliptic operator twisted by an A-module vector bundle $E$ on which the $S^{1}$ acts trivially. Let $\sigma=\sigma\left(D_{E}\right) \in K_{S^{1}}(T X, A)$ be the symbol. Then for a generator $g \in S^{1}$,

$$
\operatorname{Ind}_{S^{1}}\left(D_{E}\right)(g)=(-1)^{d}\left\{\frac{\operatorname{ch}_{g}\left(i_{g}^{*} \sigma\right)}{\operatorname{ch}_{g}\left(\lambda_{-1} N_{\mathrm{C}}\right)} \hat{A}\left(F_{g}\right)^{2}\right\}\left[T F_{g}\right] .
$$

Here $i_{g}: F_{g} \hookrightarrow X$ is the inclusion map and $K_{S^{1}}(T X, A)$ is the $K$-group of $S^{1}$-equivariant $A$-module vector bundles on $T X$ with compact support. Since $g$ is a generator of $S^{1}$, the $S^{1}$ actions on $F_{g}$ and on $A$ are trivial. One has

$$
i_{g}^{*} \sigma \in K_{S^{1}}\left(T F_{g}, A\right) \simeq K\left(T F_{g}\right) \otimes K_{0}(A) \otimes R\left(S^{1}\right) .
$$


The Chern character $\mathrm{ch}_{g}$ is given by

$$
\operatorname{ch}_{g}: \sum \alpha_{i} \otimes a_{i} \otimes \chi_{i} \rightarrow \sum \operatorname{ch} \alpha_{i} a_{i} \chi_{i}(g) \in H^{*}\left(T F_{g}, \mathbf{Q}\right) \otimes K_{0}(A)[\chi(g)],
$$

where $\alpha_{i} \in K\left(T F_{g}\right), a_{i} \in K_{0}(A)$, and $\chi_{i} \in R\left(S^{1}\right)$.

The proof of this lemma is the same as in Atiyah-Segal [6].

Now for our case $A=C^{*}(\pi)$ (the completion of $L^{1}(\pi)$ in the greatest $C^{*}$-norm, see [31]). Because the induced $S^{1}$ action is trivial on $\pi=\pi_{1}(X), S^{1}$ acts trivially on $i_{g}^{*} E$. So after reduction the local terms are also given by

$$
\prod_{m} F\left(N_{m}, t^{m}\right) \hat{A}\left(F_{g}\right) \operatorname{ch}\left(i_{g}^{*} E\right)\left[F_{g}\right],
$$

where $\operatorname{ch}\left(i_{g}^{*} E\right)$ is in the sense of [25 or 31]. On the other hand globally $\operatorname{Ind}_{S^{1}} D_{E}(g) \in K_{0}(A)\left[t, t^{-1}\right]$ by the above definition of $\operatorname{Ind}_{S^{1}} D_{E}(g)$. Both the local and the global expression are rational functions in $t$ with coefficients in $K_{0}(A)$. The same argument as in the proof of Theorem 4 gives us the required result. The only difference is that we are considering functions with coefficients in $K_{0}(A)$.

The case of elliptic genus is the same. We also omit the unpleasant detail here.

In a forthcoming paper we will give a simple proof of the Witten rigidity theorems. We would like to give the details of the above proofs for the cases of elliptic genera in that paper. Note that there exists an $S^{1}$ action on a torus which induces non-trivial action on the fundemental group. We also know that some higher $\hat{\mathfrak{A}}$-indices of a torus are non-zero. So the assumption that the $S^{1}$ action on the fundamental group is trivial is necessary in Corollary 5.

It is worthwhile to remark that the above method is simple, but it may be used to give the deep theorems in Browder-Hsiang [12], Berstein [10], who proved them in totally different ways. [12] used equivariant surgery and [10] used category theory. There are two points to be noted here. First, the method we used above also works for some vanishing theorems of secondary characteristic classes for group actions on foliations of manifolds. This requires cyclic cohomology formulation of the index theorem. Second, the twisted elliptic genera may give new invariants relating the fundamental group to the topology of a manifold.

Now, we define higher elliptic genera of a manifold $X$ with fundamental group $\pi$, as

$$
\varphi_{q}(X, a)=\left\langle\hat{A}(X) \operatorname{ch} \Theta_{q}((T X-\operatorname{dim} X) \otimes \mathbf{C}) \cup f^{*} a, X\right\rangle,
$$

where $f: X \rightarrow K(\pi, 1)$ is the induced map and $a \in H^{*}(K(\pi, 1), \mathbf{Q})$ is any element. Then a proof following Rosenberg [31] gives an easy corollary.

Corollary 6. If the strong Novikov conjecture is true for $\pi$, then for any compact smooth spin manifold $X$ with fundamental group $\pi$ and a compact connected Lie group action which induces trivial action on $\pi$, then

a) all higher $\hat{\mathfrak{A}}$-genera vanish,

b) all higher elliptic genera are rigid.

We remark that the Novikov conjecture is true for many known groups.

Following the discussion in Sect. 5 below, we can easily see that the above higher elliptic genera are sums of modular forms with different weights, with the 
highest weight $\leqq \frac{1}{2} \operatorname{dim} X$. Also, we don't need to restrict the dimension of $X$. In fact, higher elliptic genera make sense for manifolds of any dimension.

We can also define elliptic genera for families of elliptic operators, and $\mathrm{KO}$ higher elliptic genera and $\mathrm{KO}$-elliptic genera for families. In fact, we can formulate elliptic genera with values in a general $C^{*}$-algebra. This will include both the case of families of elliptic operators and the case of higher elliptic genera.

\section{On Characteristic Series of Elliptic Genera}

In this section, we will write down the characteristic series for the elliptic genera defined by Witten in [36], so that we can see the properties of elliptic genera very clearly. We use the cohomological form of the Atiyah-Singer index theorem for twisted Dirac operators. These formulas will be used to derive some properties of elliptic genera and to get some interesting corollaries in the appendix. We will also answer a question of Krichever [20]. The basic idea of this part is motivated by Brylinski [13], but the similarity of our formulas to the general Baker-Akhiezer functions in conformal field theory and integrable systems is another motivation.

Now, let us consider the Jacobi theta-functions. There are several different expressions of them; we will use the product expressions to fit our purposes

$$
\begin{aligned}
& \theta_{3}(v, \tau)=c \prod_{n=1}^{\infty}\left(1+q^{n-\frac{1}{2}} e^{2 \pi i v}\right) \prod_{n=1}^{\infty}\left(1+q^{n-\frac{1}{2}} e^{-2 \pi i v}\right), \\
& \theta_{2}(v, \tau)=c \prod_{n=1}^{\infty}\left(1-q^{n-\frac{1}{2}} e^{2 \pi i v}\right) \prod_{n=1}^{\infty}\left(1-q^{n-\frac{1}{2}} e^{-2 \pi i v}\right), \\
& \theta_{1}(v, \tau)=c q^{1 / 8} e^{\pi i v} \prod_{n=1}^{\infty}\left(1+q^{n} e^{2 \pi i v}\right) \prod_{n=0}^{\infty}\left(1+q^{n-1} e^{-2 \pi i v}\right), \\
& \theta(v, \tau)=c q^{1 / 8} 2 \sin \pi v \prod_{n=1}^{\infty}\left(1-q^{n} e^{2 \pi i v}\right) \prod_{n=1}^{\infty}\left(1-q^{n} e^{-2 \pi i v}\right),
\end{aligned}
$$

where $c=\prod_{n=1}^{\infty}\left(1-q^{n}\right), q=e^{2 \pi i \tau}$, and $\operatorname{Im} \tau>0$. Note the slight difference between the $q$ here and the $q$ in [15]. We also have the following transformation formulas for theta-functions

$$
\begin{aligned}
\sqrt{\frac{\tau}{i}} \theta_{3}(v, \tau) & =e^{-\left(\pi i v^{2}\right) / \tau} \theta_{3}\left(\frac{v}{\tau},-\frac{1}{\tau}\right), \\
\sqrt{\frac{\tau}{i}} \theta_{2}(v, \tau) & =e^{-\left(\pi i v^{2}\right) / \tau} \theta_{1}\left(\frac{v}{\tau},-\frac{1}{\tau}\right), \\
\sqrt{\frac{\tau}{i}} \theta_{1}(v, \tau) & =e^{-\left(\pi i v^{2}\right) / \tau} \theta_{2}\left(\frac{v}{\tau},-\frac{1}{\tau}\right), \\
\sqrt{\frac{\tau}{i}} \theta(v, \tau) & =i e^{-\left(\pi i v^{2}\right) / \tau} \theta\left(\frac{v}{\tau},-\frac{1}{\tau}\right),
\end{aligned}
$$


and at $v \stackrel{\prime}{=} 0$, we have

$$
\begin{aligned}
\theta_{3}(0, \tau) & =\sqrt{\frac{i}{\tau}} \theta_{3}\left(0,-\frac{1}{\tau}\right), \\
\theta_{2}(0, \tau) & =\sqrt{\frac{i}{\tau}} \theta_{1}\left(0,-\frac{1}{\tau}\right), \\
\theta_{1}(0, \tau) & =\sqrt{\frac{i}{\tau}} \theta_{2}\left(0,-\frac{1}{\tau}\right), \\
\sqrt{\frac{\tau}{i}} \theta^{\prime}(0, \tau) & =\frac{i}{\tau} \theta^{\prime}\left(0,-\frac{1}{\tau}\right),
\end{aligned}
$$

where $\theta^{\prime}(0, \tau)$ means taking the derivative with respect to $v$.

We would like to write down more formulas to show that some properties of elliptic genera easily follow from these formulas:

$$
\begin{aligned}
\theta(v+1, \tau) & =-\theta(v, \tau), \\
\theta(v, \tau) & =-\theta(-v, \tau), \\
\theta(v+\tau, \tau) & =-q^{-\frac{1}{2}} e^{2 \pi i v} \theta(v, \tau) .
\end{aligned}
$$

Similar formulas for $\theta_{1}, \theta_{2}, \theta_{3}$ are also true (see [15]).

We write the formal Chern roots of $T X \otimes \mathbf{C}$, the complexified tangent bundle of a compact smooth manifold $X$ of dimension $2 k$, as

$$
2 \pi i \beta_{1},-2 \pi i \beta_{1}, \ldots 2 \pi i \beta_{k},-2 \pi i \beta_{k} .
$$

Recall that one calls a formal power series $f(v)$ a characteristic series of a genus $\phi$ on a manifold $X$, if

$$
\phi(X)=\left\langle f\left(\beta_{1}\right) \ldots f\left(\beta_{k}\right), X\right\rangle .
$$

Then we have

Theorem 4. For a dimension $2 k$ compact smooth spin manifold $X$, we have the following correspondences of characteristic series to elliptic genera:

$$
\begin{gathered}
2 \pi i v \frac{\theta_{1}(v, \tau)}{\theta(v, \tau)} \leftrightarrow \operatorname{Ind}\left(D \otimes \Delta(X) \otimes \Theta_{q}^{\prime}(T X)\right), \\
2 \pi i v \frac{\theta_{2}(v, \tau)}{\theta(v, \tau)} \leftrightarrow q^{-k / 8} \operatorname{Ind}\left(D \otimes \Theta_{q}(T X)\right), \\
2 \pi i v \frac{\theta_{3}(v, \tau)}{\theta(v, \tau)} \leftrightarrow q^{-k / 8} \operatorname{Ind}\left(D \otimes \Theta_{-q}(T X)\right), \\
2 \pi i v \frac{\theta_{2}(v, 2 \tau)}{\theta(v, 2 \tau)} \leftrightarrow q^{-k / 4} \operatorname{Ind}\left(D \otimes \Theta_{q}^{*}(T X)\right),
\end{gathered}
$$

and

$$
2 i v \frac{\theta_{j}(v, \tau)}{\theta(v, \tau)} \frac{\theta^{\prime}(0, \tau)}{\theta_{j}(0, \tau)}
$$


for $j=1,2,3$ together with

$$
2 i v \frac{\theta_{2}(v, 2 \tau)}{\theta(v, 2 \tau)} \frac{\theta^{\prime}(0,2 \tau)}{\theta_{2}(0,2 \tau)}
$$

exactly correspond to the virtual genera, i.e. we replace $T X$ by $T X-\operatorname{dim} X$ and omit $q^{-k / 8}$ or $q^{-k / 4}$ in above correspondences.

Remarks. (i): If we replace $\tau$ by $2 \tau$ or $\tau+1$ in the expression of the characteristic series of $\operatorname{Ind}\left(D \otimes \Theta_{q}(T X-\operatorname{dim} X)\right)$, we get the characteristic series of $\operatorname{Ind}\left(D \otimes \Theta_{q}^{*}(T X-\operatorname{dim} X)\right)$ or $\operatorname{Ind}\left(D \otimes \Theta_{-q}(T X-\operatorname{dim} X)\right)$ respectively. Similarly for the nonvirtual elliptic genera.

(ii): It is interesting to note that even the anomaly factor $q^{-k / 8}$ in Witten's formulas in [35] and [36], which come from renomalization of the Feynman path integral perfectly fits our theta-function expressions. For compact almost complex manifolds with $c_{1} \equiv 0(\bmod N)$ we can express the characteristic series of the elliptic genera as

$$
2 \pi i x \frac{\theta(x+\alpha, \tau)}{\theta(x, \tau)}
$$

or

$$
2 i x \frac{\theta(x+\alpha, \tau)}{\theta(x, \tau)} \frac{\theta^{\prime}(0, \tau)}{\theta(\alpha, \tau)}
$$

with $e^{2 \pi i \alpha}$ an $N^{\text {th }}$ root of unity. The transformations of these expressions by the elements in $S L_{2}(\mathbf{Z})$ give all of the elliptic genera on complex manifolds with $c_{1} \equiv 0(\bmod N)$. We omit the details here. have

(iii): From our formulas and transformation formulas of theta-functions, we

$$
\frac{\theta_{2}(v, \tau)}{\theta(v, \tau)}=\frac{1}{i} \frac{\theta_{1}\left(\frac{v}{\tau},-\frac{1}{\tau}\right)}{\theta\left(\frac{v}{\tau},-\frac{1}{\tau}\right)}, \frac{\theta_{2}(v, \tau+1)}{\theta(v, \tau+1)}=\chi_{8} \frac{\theta_{3}(v, \tau)}{\theta(v, \tau)}
$$

and

$$
\frac{\theta_{3}(v, \tau)}{\theta(v, \tau)}=\frac{1}{i} \frac{\theta_{3}\left(\frac{v}{\tau},-\frac{1}{\tau}\right)}{\theta\left(\frac{v}{\tau},-\frac{1}{\tau}\right)}, \frac{\theta_{1}(v, \tau+1)}{\theta(v, \tau+1)}=\chi_{8}^{\prime} \frac{\theta_{1}(v, \tau)}{\theta(v, \tau)}
$$

with $\chi_{8}, \chi_{8}^{\prime} 8^{\text {th }}$ roots of unity. Furthermore

$$
\begin{aligned}
& \frac{\theta_{2}(v, \tau)}{\theta(v, \tau)} \frac{\theta^{\prime}(0, \tau)}{\theta_{2}(0, \tau)}=\frac{1}{\tau} \frac{\theta_{1}\left(\frac{v}{\tau},-\frac{1}{\tau}\right)}{\theta\left(\frac{v}{\tau},-\frac{1}{\tau}\right)} \frac{\theta^{\prime}\left(0,-\frac{1}{\tau}\right)}{\theta_{1}\left(0,-\frac{1}{\tau}\right)}, \\
& \frac{\theta_{3}(v, \tau)}{\theta(v, \tau)} \frac{\theta^{\prime}(0, \tau)}{\theta_{3}(v, \tau)}=\frac{\theta_{2}(v, \tau+1)}{\theta(v, \tau+1)} \frac{\theta^{\prime}(0, \tau+1)}{\theta_{2}(v, \tau+1)}
\end{aligned}
$$


and

$$
\begin{aligned}
& \frac{\theta_{3}(v, \tau)}{\theta(v, \tau)} \frac{\theta^{\prime}(0, \tau)}{\theta_{3}(0, \tau)}=\frac{1}{\tau} \frac{\theta_{3}\left(\frac{v}{\tau},-\frac{1}{\tau}\right)}{\theta\left(\frac{v}{\tau},-\frac{1}{\tau}\right)} \frac{\theta^{\prime}\left(0,-\frac{1}{\tau}\right)}{\theta_{3}\left(0,-\frac{1}{\tau}\right)}, \\
& \frac{\theta_{1}(v, \tau)}{\theta(v, \tau)} \frac{\theta^{\prime}(0, \tau)}{\theta_{1}(v, \tau)}=\frac{\theta_{1}(v, \tau+1)}{\theta(v, \tau+1)} \frac{\theta^{\prime}(0, \tau+1)}{\theta_{1}(v, \tau+1)} .
\end{aligned}
$$

Corollary 7. Let $X$ be a compact smooth spin manifold of dimension $2 k$. Then

i) the following elliptic genera are modular forms of weight $k$ with integral Fourier coefficients over the corresponding modular groups:

a) Ind $\left(D \otimes \Delta(X) \otimes \Theta_{q}^{\prime}(T X-\operatorname{dim} X)\right)$ over $\Gamma_{0}(2)$;

b) Ind $\left(D \otimes \Theta_{q}(T X-\operatorname{dim} X)\right)$ over $\Gamma^{0}(2)$;

c) $\operatorname{Ind}\left(D \otimes \Theta_{-q}(T X-\operatorname{dim} X)\right)$ over $\Gamma_{\theta}$;

d) $\operatorname{Ind}\left(D \otimes \Theta_{q}^{*}(T X-\operatorname{dim} X)\right)$ over $\Gamma_{0}(2)$.

ii) the following elliptic genera are (twisted) automorphic functions with integral Fourier coefficients over the corresponding modular groups:

a) $\operatorname{Ind}\left(D \otimes \Delta(X) \otimes \Theta_{q}^{\prime}(T X)\right)$ over $\Gamma_{0}(2)$;

b) $q^{-k / 8} \operatorname{Ind}\left(D \otimes \Theta_{q}(T X)\right)$ over $\Gamma^{0}(2)$;

c) $q^{-k / 8} \operatorname{Ind}\left(D \otimes \Theta_{-q}(T X)\right)$ over $\Gamma_{\theta}$

d) $q^{-k / 4} \operatorname{Ind}\left(D \otimes \Theta_{q}^{*}(T X)\right)$ over $\Gamma_{0}(2)$.

In fact since

$$
T: \tau \mapsto \tau+1, \quad S: \tau \mapsto-\frac{1}{\tau}
$$

generate $S L_{2}(\mathbf{Z})$ and $T, S T^{2} S T$ generate $\Gamma_{0}(2)$, it is easy to get the modular property of $\operatorname{Ind}\left(D \otimes \Delta(X) \otimes \Theta_{q}^{\prime}(T X-\operatorname{dim} X)\right)$ over $\Gamma_{0}(2)$ by using the above formulas, similarly for the other elliptic genera. These formula can be used to prove the rigidity of $D \otimes \Theta_{q}(T X)$ or $D \otimes \Theta_{-q}(T X)$ from that of $D \otimes \Delta(X) \otimes \Theta_{q}^{\prime}(T X)$. (See the discussion in the appendix.)

If we note that $\Gamma^{0}(2)$ and $\Gamma_{\theta}$ are conjugate to $\Gamma_{0}(2)$ by $S$ and $S T$ respectively, the modular properties of the other elliptic genera also follow easily from the above formulas. In fact the other elliptic genera are conjugate to $\operatorname{Ind}\left(D \otimes \Delta(X) \otimes \Theta_{q}^{\prime}(T X-\operatorname{dim} X)\right.$ by $S$ and $S T$. This observation is due to Brylinski. It is also amusing to prove Corollary 7 directly by using the transformation formulas of theta-functions.

The above equalities mean that these three virtual elliptic genera patch together to give a section of a holomorphic line bundle on an elliptic modular surface. They correspond to the three cusps of this elliptic modular surface. The nonvirtual elliptic genera patch to a meromorphic function on this elliptic modular surface. Many of the properties of these elliptic genera can be derived from the geometry of this elliptic modular surface. We will come back to this point in a forthcoming paper.

Now we consider Krichever's genera in [20], for which the characteristic series are given by

$$
\hat{\Phi}\left(x, z, k_{0}\right)=\frac{\sigma(z-x)}{\sigma(x) \sigma(z)} e^{\zeta(z) x-k_{0} x}
$$


where $\sigma(z), \zeta(z)$ are Weierstrass functions (see [15]). Following [20], we take $x$ as variable, $z$ as parameter. Concerning Krichever's equation, we have

Theorem 5. For SU-manifold $X, 2 i x \hat{\Phi}\left(x, z, k_{0}\right)$ is the characteristic series of a twisted Dirac operator $D \otimes R_{q}(T X-\operatorname{dim} X)$, where

$$
\begin{aligned}
R_{q}(T X-\operatorname{dim} X)= & \underset{n \geqq 0}{\otimes} \Lambda_{-y q^{n}}\left(T X^{*}-\operatorname{dim} X\right) \underset{n \geqq 1}{\otimes} \Lambda_{-y^{-1} q^{n}}(T X-\operatorname{dim} X) \\
& \bigotimes_{n \geqq 1}^{\otimes} S_{q^{n}}\left(T X^{*}-\operatorname{dim} X\right) \underset{n \geqq 1}{\otimes} S_{q^{n}}(T X-\operatorname{dim} X)
\end{aligned}
$$

with $y=e^{2 \pi i z}$.

Proof. By $S U$-manifolds, we mean the structure groups of their stable tangent bundles can be reduced to special unitary groups. Now we consider the formula in [15]

$$
\sigma(u)=\theta(u, \tau) \frac{1}{\theta^{\prime}(0, \tau)} e^{\eta_{1} u^{2}}
$$

where $\{n+m \tau\}$ is the lattice of the elliptic curve, and $\eta_{1}=\zeta\left(\frac{1}{2}\right)$. Then we have

$$
\hat{\Phi}\left(x, z, k_{0}\right)=\frac{\theta(z-x, \tau)}{\theta(z, \tau) \theta(x, \tau)} e^{\left(\zeta(z)-2 \eta_{1} z-k_{0}\right) x} \theta^{\prime}(0, \tau) .
$$

Since for $S U$-manifolds, the term $e^{\left(\zeta(z)-2 \eta_{1} z-k_{0}+\frac{1}{2}\right) x}$ can be omitted in computing genera, we can apply the Atiyah-Singer index theorem to $D \otimes R_{q}(T X-\operatorname{dim} X)$ to get our result.

If $\zeta(z)-2 \eta_{1} z-k_{0}+\frac{1}{2}$ is an integer $k$ for some $z$, then $2 i x \hat{\Phi}\left(x, z, k_{0}\right)$ is the characteristic series of $D \otimes L^{k} \otimes R_{q}(T X-\operatorname{dim} X)$, where $L=\operatorname{det} T X$, even without the assumption that the manifold is $S U$. If $\zeta(z)-2 \eta_{1} z-k_{0}$ is an integer and the manifold has an almost complex structure, we can express $2 i x \hat{\Phi}\left(x, z, k_{0}\right)$ as the characteristic series of a twisted $\bar{\partial}$-operator. It is interesting to note that in these cases the genera are rigid (see [20]). But we do not know whether these genera are still rigid without the assumption that $\zeta(z)-2 \eta_{1} z-k_{0}$ or $\zeta(z)-2 \eta_{1} z-k_{0}+\frac{1}{2}$ are integers. Because in this case, it seems to be impossible to express them as the indices of elliptic operators. Note the interesting similarity between $\hat{\Phi}\left(x, z, k_{0}\right)$ and $F_{\tau}(u, v)$ in [38] where Zagier used this function to get the generating function for the Fourier coefficients of modular forms.

These genera are more general than those of Hirzebruch's. In [17] $y$ is only a root of unity; here we get a continuous family of elliptic genera. The interesting point is that for those manifolds on which they can be written as the indices of elliptic operators these genera are still rigid by Krichever [20]. Of course the rigidity can also be proved by transfer formula in index theory. In fact, Krichever used transfer formula in cobordism theory.

It is interesting to notice that so far all of the genera which have rigidity properties can be written as the indices of elliptic operators. This should be related to the homotopy invariance of the indices of elliptic operators. Recently, Morava and Shimizu [26] have constructed some topological generalization of the elliptic genera. It is quite difficult to see whether these new genera are rigid or whether they can be written as the indices of elliptic operators. From the point of view of physics, only the rigid genera are interesting (see Witten [36]). This should be an interesting problem for further study. 
Krichever's formula come from KP-equations for elliptic curves. This may indicate that it is possible to get more general elliptic genera and to give geometric explanations of elliptic cohomology from integrable systems.

\section{Appendix}

In this appendix, we collect some known facts in the theory of elliptic genera for the convenience of readers. We also give some general discussions about elliptic genera.

We first discuss dimensions $8 k$ and $8 k+4$ cases. In these cases elliptic genera are given by the characteristic series in Sect. 5. The modular properties of elliptic genera follow easily from the formulas there. In fact, Zagier [37] gives some of the equivalence relations in elliptic genera which were first discovered by Landweber-Stong, Ochanine and Witten (see [21]). We take $\phi_{q}(X)=\operatorname{Ind}\left(D \otimes \Theta_{q}^{*}(T X-\operatorname{dim} X)\right)$ as an example. Here recall

$$
\Theta_{q}^{*}(E)=\bigotimes_{n \geqq 1}\left(\Lambda_{-q^{2 n-1}} E \otimes S_{q^{2 n}} E\right) .
$$

Then everything is compatible with [37 and 28].

First we have

where

$$
g(x)=\int_{0}^{x} \frac{d t}{\sqrt{1-2 \delta t^{2}+\varepsilon t^{4}}}
$$

$$
g(x)=\sum_{n=0}^{\infty} \phi_{q}\left(\mathbf{C} P^{2 n}\right) \frac{x^{2 n+1}}{2 n+1}
$$

is called the logarithm of the formal group law of $\phi_{q}$ and

$$
f(u, v)=g^{-1}(g(u)+g(v))
$$

is called the formal group law of $\phi_{q}$. Here $\varepsilon$ is the same as the $\varepsilon_{0}$ in Sect. 3 and $8 \delta=\delta_{0}$. The characteristic series of $\phi_{q}$ is also equal to $x / g^{-1}(x)$ which can be derived from the Lagrange formula in elementary function theory. So by Theorem 5 in Sect. 5 ,

$$
g^{-1}(x)=\pi \frac{\theta(x, 2 \tau) \theta_{2}(0,2 \tau)}{\theta_{2}(x, 2 \tau) \theta^{\prime}(0,2 \tau)}
$$

From Zagier [37], (or from Sect. 5 of this paper) we also know that for any compact spin manifold $M, \phi_{q}(M)$ is a modular form over $\Gamma_{0}(2)$.

Theorem $\mathbf{A 1}([21]) . \phi_{q} \Omega_{*}^{\text {spin }}=\mathbf{Z}\left[16 \delta,(8 \delta)^{2}, \varepsilon\right]$.

This $\phi_{q}$ kills the torsion elements in $\Omega_{*}^{\text {spin }}$ as well as $8 k+1$ and $8 k+2$ dimensional elements. Ochanine refined this result. He defined $\mathrm{KO}_{*}$-valued elliptic genera $\beta_{q}$ and obtained.

Theorem A2 ([29]).

1) If $n=4 s, \beta_{q}\left(\Omega_{n}^{\text {spin }}\right)$ is the set of all modular forms of degree $n$ and weights $2 s$ over $K O_{*}$. 
2) If $n=8 m+r,(r=1,2)$, then $\beta_{q}\left(\Omega_{n}^{\text {spin }}\right)$ is the set of all modular forms of degree $n$ and filtration $\leqq 4 m$ over $K O_{*}$. and $\varepsilon$.

3) $\beta_{q}\left(\Omega_{*}^{\text {spin }}\right)$ is the subring of modular forms over $K O_{*}$ generated by $\eta, \omega \delta_{0}, \delta_{0}^{2}$

For the meanings of weight and filtration, we refer to his original paper [29]. $\beta_{q}(X)$ is a lift of $\phi_{q}(X)$ to $K O_{*}$ when $\operatorname{dim} X=4 s$.

Hirzebruch and Slodowy [17] proved that for a compact spin homogeneous manifold $M$

$$
\phi_{q}(M)=\left\{\begin{array}{ll}
0 & \operatorname{dim} M=4 k \\
c \cdot \varepsilon^{k} & \operatorname{dim} M=8 k
\end{array},\right.
$$

where $c$ is the signature of $M$. This extends Landweber-Revenel-Stong's computations for $\mathbf{H} P^{n}$. A pure cobordism proof of Hirzebruch and Slodowy's results may be very interesting. We do not know any similar results about the mod 2 elliptic genera for compact spin homogeneous manifolds of dimension $8 k+1$ and $8 k+2$. We like to point out that the vanishing theorems of characteristic numbers in Landweber-Stong and Ochanine (see [28]) can also be proved by Bott residue formula by constructing explicit holomorphic vector field on $\mathbf{C} P^{2 n+1}$ (see [23]). This proof is very elementary.

The elliptic cohomology

$$
\mathrm{Ell}^{*}(X)=\Omega_{*}^{S o}(X) \otimes \mathbf{Z}\left[\frac{1}{2}\right]\left[\delta, \varepsilon,\left(\delta^{2}-\varepsilon\right)^{-1}\right]
$$

was constructed by Landweber-Ravenel-Stong (see [21]). It is interesting to consider the following generalized Chern character:

$$
\operatorname{ch}_{E}: \operatorname{Ell}^{*}(X) \rightarrow H^{*}\left(X, \mathbf{Q}\left[\delta, \varepsilon,\left(\delta^{2}-\varepsilon\right)^{-1}\right]\right)
$$

which was developed by Buhstaber [14] to extend the usual Chern character from $K$-theory to de Rham cohomology.

Generalized cohomology theory tells us that $\operatorname{Ell}^{*}\left(\mathbf{C} P^{\infty}\right)=\operatorname{Ell} *(p t)[u]$, where $u$ is the Chern class of the universal line bundle over $\mathbf{C} P^{\infty}$ in elliptic cohomology. Similarly $H^{*}\left(\mathbf{C} P^{\infty}, \mathbf{Q}\right)=\mathbf{Q}[2 \pi i x]$ with $2 \pi i x$ the Chern class of the universal line bundle in de Rham cohomology. For $\phi_{q}$ we have

$$
\begin{aligned}
\operatorname{ch}_{E}: \mathrm{Ell}^{*}(p t)[u] & \rightarrow \mathbf{Q}\left[\delta, \varepsilon,\left(\delta^{2}-\varepsilon\right)^{-1}\right][2 \pi i x] \\
u & \mapsto \pi \frac{\theta(x, 2 \tau) \theta_{2}(0,2 \tau)}{\theta_{2}(x, 2 \tau) \theta^{\prime}(0,2 \tau)} .
\end{aligned}
$$

For $\operatorname{Ind}\left(D \otimes \Delta(T X) \otimes \Theta_{q}^{\prime}(T X-\operatorname{dim} X)\right)$, we can get

$$
\operatorname{ch}_{E}(u)=\pi \frac{\theta(x, \tau) \theta_{1}(0, \tau)}{\theta_{1}(x, \tau) \theta^{\prime}(0, \tau)}
$$

Similar formulas also hold for the other elliptic genera in Sect. 5. So $\mathrm{ch}_{E}$ is quite similar to some partition function in holomorphic conformal field theory. We can also lift $\mathrm{ch}_{E}$ to $K^{*}\left(\mathbf{C} P^{\infty}\right) \otimes \mathbf{Q}$ to get similar formulas. 
Now let us briefly sketch an interesting corollary of the theta-function expressions of the elliptic genera in Sect. 5. This is the "duality" property between the rigidities of different elliptic genera. We consider the virtual versions and only give a sketch here. The detailed discussion as well as an algebro-geometric explanation of the Witten rigidity theorems will be given in a forthcoming paper.

Let $X$ be a compact smooth spin manifold with an $S^{1}$ action. Let $g=e^{2 \pi i t} \in S^{1}$ be a generator of the action group. Let $\{p\} \subset X$ be the fixed points of $g$. For simplicity we only consider isolated fixed point case. Let

$$
\left.T X\right|_{p}=E_{1} \oplus \cdots \oplus E_{k}, \quad k=\frac{1}{2} \operatorname{dim} X
$$

be the decomposition of the tangent bundle into the $S^{1}$-invariant 2-planes when restricted to the fixed points. $g$ acts on $E_{j}$ by multiplication by $e^{2 \pi i m_{j} t}$ with $m_{j} \in \mathbf{Z}$. Choose the orientation of $E_{j}$ compatibility with the orientation of $X$. Let

$$
\begin{gathered}
F_{d_{s}}(t, \tau)=\pi^{-k} \sum_{p} \prod_{j=1}^{k}\left(\frac{\theta_{1}\left(m_{j} t, \tau\right) \theta^{\prime}(0, \tau)}{\theta\left(m_{j} t, \tau\right) \theta_{1}(0, \tau)}\right), \\
F_{D}(t, \tau)=\pi^{-k} \sum_{p} \prod_{j=1}^{k}\left(\frac{\theta_{2}\left(m_{j} t, \tau\right) \theta^{\prime}(0, \tau)}{\theta\left(m_{j} t, \tau\right) \theta_{2}(0, \tau)}\right), \\
F_{-D}(t, \tau)=\pi^{-k} \sum_{p} \prod_{j=1}^{k}\left(\frac{\theta_{3}\left(m_{j} t, \tau\right) \theta^{\prime}(0, \tau)}{\theta\left(m_{j} t, \tau\right) \theta_{3}(0, \tau)}\right),
\end{gathered}
$$

where $d_{s}=D \otimes \Delta(T)$ denotes the signature operator and $D$ denotes the pure Dirac operator.

Then the Lefschetz fixed point formula for $D \otimes \Theta_{q}(T X-\operatorname{dim} X)$, $D \otimes \Theta_{-q}(T X-\operatorname{dim} X)$ and $d_{s} \otimes \Theta_{q}^{\prime}(T X-\operatorname{dim} X)$ tell us that

$$
\begin{gathered}
F_{d_{s}}(t, \tau)=\text { the Lefschetz number of } d_{s} \otimes \Theta^{\prime}(T X-\operatorname{dim} X), \\
F_{D}(t, \tau)=\text { the Lefschetz number of } D \otimes \Theta_{q}(T X-\operatorname{dim} X), \\
\left.F_{-D}(t, \tau)=\text { the Lefschetz number of } D \otimes \Theta_{-q}(T X S-\operatorname{dim} X)\right) .
\end{gathered}
$$

Here we use the same notations as in Sect. 5. It is easy to verify that $F_{d_{s}}(t, \tau)$, $F_{D}(t, \tau)$ and $F_{-D}(t, \tau)$ are elliptic functions in $t$ on $\mathbf{C}^{*} / q^{2}$. In fact, this is a simple corollary of the transformation formulas of theta-functions.

The rigidity theorems are equivalent to that $F_{-D}(t, \tau), F_{D}(t, \tau)$ and $F_{d_{s}}(t, \tau)$ are independent of $t$.

Corollary A1. For a compact smooth spin manifold $X$ the rigidity of one of the above three elliptic genera implies the rigidities of the other two.

Proof. By the transformation formulas of theta-functions, we have

$$
F_{d_{s}}\left(\frac{t}{\tau},-\frac{1}{\tau}\right)=F_{D}(t, \tau) \cdot \tau^{k}
$$

and

$$
F_{D}(t, \tau+1)=F_{-D}(t, \tau)
$$


If $X$ is spin $F_{d_{s}}(t, \tau)$ independent of $t$ the above formulas tell us that $F_{D}(t, \tau)$ and $F_{-D}(t, \tau)$ are also independent of $t$.

We like to call this corollary the "duality" property of elliptic genera.

Consider the first terms in the $q$-expansions of $F_{d_{s}}(t, \tau)$ and $F_{D}(t, \tau)$ which is the Lefschetz number of $d_{s}$ and $D$ respectively. Then the above corollary means that $d_{s}$ and $D$ are also "dual" to each other.

The general fixed point case of this corollary can also be proved in this way. We will give the details in a separate paper in which we will study the elliptic genera twisted by general vector bundles and elliptic genera on complex manifolds. In that paper we will relate all of the properties of the elliptic genera to the geometry of elliptic modular surfaces. The theta-function expressions of the elliptic genera will play important role in that paper.

\section{References}

1. Atiyah, M.F.: Collected Works. Oxford: Oxford Science Publication

2. Atiyah, M.F.: Algebraic Topology and Elliptic Operators. In [1], Vol. 3, pp. 57-70

3. Atiyah, M.F.: Vector Fields on Manifolds. In [1], Vol. 2, pp. 727-749

4. Atiyah, M.F.: Bott, R., Shapiro, A.: Clifford Modules. In [1], Vol. 2, pp. 299-335

5. Atiyah, M.F., Hirzebruch, F.: Spin Manifolds and Group Actions. In [1], Vol. 3, pp. 417-429

6. Atiyah, M.F., Segal, G.: The Index of Elliptic Operators. In [1], Vol. 3, pp. 224-237

7. Atiyah, M.F., Singer, I.M.: The Index of Elliptic Operators V. In [1], Vol. 3, pp. 323-347

8. Atiyah, M.F., Patodi, V.K., Singer, I.M.: Spectral Asymmetry and Riemannian Geometry I, II. In [1], Vol. 4, pp. 87-137

9. Bendersky, M.: Cobordism Span of a Manifold and Elliptic Genera. Math. Z. 202, 483-492 (1989)

10. Berstein, I.: On Covering Space and Lie Group Actions. Algebraic Topology in honor of Peter Hilton, pp. 11-13

11. Bott, R., Taubes, C.: On Rigidity Theorem of Witten. J. Am. Math. Soc. 2, 137-186 (1989)

12. Browder, W., Hsiang, W.C.: G-Actions and The Fundamental Groups. Invent. Math. 69, 411-424 (1982)

13. Brylinski, J.-L.: Remark on Witten's Modular Forms. Proc. Am. Math. Soc. 105, 773-775 (1989)

14. Buhstaber, V.M.: The Chern-Dold Character in Cobordisms I. Mat. Sbornik Tom 83, 573-594 (1970)

15. Chandrasekharan, K.: Elliptic Functions. Berlin Heidelberg New York: Springer

16. Hirzebruch, F.: Elliptic Genera of Level N for Complex Manifolds. Differential Geometrical Methods in Theoretical Physics, pp. 37-63. Dordrecht: Kluwer 1988

17. Hirzebruch, F., Slodowy, P.: Ellptic Genera, Involutions and Homogeneous Manifolds. Geom. Dedicate. 35, 309-343 (1990)

18. Hopkins, M.T., Hovey, M.A.: Spin Cobordism Determines Real K-Theory. Preprint 1990

19. Hovey, M.A.: Spin Bordism and Elliptic Homology. Preprint 1991

20. Krichever, I.: Generalized Elliptic Genera and Baker-Akhiezer Functions. Math. Notes 47, 132-142 (1990)

21. Landweber, P.S.: Elliptic Curves and Modular Forms in Algebraic Topology. Lecture Notes in Math. vol 1326, Berlin Heidelberg New York: Springer

22. Lawson, H.B. Jr., Yau, S.-T.: Scalar Curvature, Nonabelian Group Actions and Degree of Symmetry of Exotic Spheres. Commun. Math. Helv. 49, 232-244 (1974)

23. Liu, K.F.: Holomorphic Vector Fields on Complex Manifolds. Preprint 1987

24. Milnor, J.: Remarks Concening Spin Manifolds, pp. 55-62. Symposium in Honor of Marston Morse

25. Mishchenko, A.S., Fomenko, A.T.: The Index of Elliptic Operators over C*-Algebras. Math. USSR-Izv. 15, 87-112 (1980) 
26. Moravd, J., Shimizu, Y.: A Topological Generalization of The Elliptic Genus. Preprint 1990

27. Moriyoshi, H.: Secondary Characteristic Numbers and Locally Free $S^{1}$-Actions. Geometric and Topological Invariants of Ellptic Operators. Contemp. Math. 105, 119-144 (1990)

28. Ochanine, S.: Sur Les Genres Multiplicatifs Definis Par Des Integrales Elliptiques. Topology 26, 143-151 (1987)

29. Ochanine, S.: Modular Forms over $\mathrm{KO}_{*}$ and The Brown-Kervaire Invariant. Math. Z. 206, 277-291 (1991)

30. Rubinsztein, R.: An Analytic Formula for The Kervaire Invariant of some ( $8 k+2)$-Dimensional Spin Manifolds. Preprint 1988

31. Rosenberg, J.: $C^{*}$-Algebras, Positive Scalar Curvatures and Novikov Conjecture. IHES 58, 409-424 (1983)

32. Serre, J.-P.: Formes Modulaires et Fonctions Zeta (modp). Modular Functions of One Variable III, Lecture Note in Math. vol 350, pp. 191-268. Berlin Heidelberg New York: Springer

33. Stong, R.: Notes on Cobordism Theory. Princeton, NJ: Princeton Univ. Press (1968)

34. Stolz, S.: Simply Connected Manifolds of Positive Scalar Curvature. Preprint 1991

35. Witten, E.: Elliptic Genera and Quantum Field Theory. Commun. Math. Physics 109, 525-536 (1987)

36. Witten, E.: The Index of the Dirac Operator in Loop Space. In [12], pp. 161-181

37. Zagier, D.: On the Landweber-Stong Elliptic Genera. In [12], pp. 216-224

38. Zagier, D.: Periods of Modular Forms and Jacobi Theta Functions. Preprint 1992

Communicated by S.-T. Yau

Note added in proof. After the author has submitted this paper, he was able to give a simple proof of the written rigidity theorems by using theta-functions. The same method can be used to prove more general rigidity theorems and to construct new elliptic genera, especially elliptic genera of level 1 . 
